\title{
Responding with hand and foot: The right/left prevalence in spatial compatibility is still present
}

\author{
ROBERTO NICOLETTI and CARLO UMILTA \\ Istituto di Psicologia, Università di Padova, Padova, Italy \\ and Istituto di Fisiologia Umana, Università di Parma, Parma, Italy
}

\begin{abstract}
Three experiments were undertaken to determine why right/left locational cues are superior to above/below cues in spatial compatibility. In Experiment 1, subjects responded with either the right or the left hand and the locational cues available involved both the right/left and above/below dimensions. The procedure was such as to allow the locational cues to vary independently and orthogonally. The results showed reliable compatibility effects in both dimensions, but the effect in the right/left dimension was stronger. Experiment 2, in which only above/below cues were present and the subjects responded with either hand or foot, showed a clear-cut above/below compatibility effect. Experiment 3 replicated Experiment 1, except that the hand and foot were used for responding. The results showed a spatial compatibility effect for the right/left dimension and no effect for the above/below dimension. The findings are discussed with reference to the explanations put forward to account for the prevalence of the right/left dimension in spatial compatibility. A tentative explanation is proposed in terms of a propensity to allocate attention to right/left locational cues at the expense of above/below cues.
\end{abstract}

In the spatial-compatibility paradigm (see, e.g., Simon, 1969), the position of the stimulus indicates the position of the correct response. For example, the right-side stimulus commands a response with the right-side (compatible condition) or the left-side (incompatible condition) key. Similarly, the top stimulus commands a response with either the top or the bottom key. A previous study (Nicoletti \& Umiltà, 1984) showed that in such choice reaction time (RT) tasks, when both right/left and above/below cues were simultaneously available to subjects for mapping stimuli to responses, spatial compatibility, though present for both dimensions, was much stronger for the right/left one.

This finding was somewhat unexpected because it did not seem to be in accordance with the well-known right/left confusion effect (see, e.g., Corballis \& Beale, 1976) and the results of those studies (Farrell, 1979; Maki, Grandy, \& Hauge, 1979; Maki, Maki, \& Marsh, 1977; Sholl \& Egeth, 1981) that had shown that locational discriminations were more difficult in the right/left than in the above/below dimension. However, the inconsistency was thought to be more apparent than real because the right/left confusion effect is known to depend on the use of verbal codes (see, e.g., Corballis \& Beale, 1976; Sholl \& Egeth, 1981), whereas the right/left prevalence in spa-

\footnotetext{
This research was supported by funds from the Consiglio Nazionale delle Ricerche and the Ministero della Pubblica Istruzione. The authors would like to thank Professor G. Berlucchi for helpful discussion of the experiments. Requests for reprints should be sent to: Carlo Umiltà, Istituto di Fisiologia Umana, Università di Parma, via A. Gramsci, 14, 43100 Parma, Italy.
}

tial compatibility might be attributable to a sort of midline barrier (Bruner, 1971) that would render stimulusresponse ( $\mathrm{S}-\mathrm{R})$ pairings on the same side of the body easier to process than those that cross the midline (Nicoletti \& Umiltà, 1984). In the right/left dimension, compatible S-R pairings do not require the crossing of the midline barrier, whereas incompatible ones do. Therefore, compatibility is stronger in the right/left dimension than in the above/below dimension, where neither compatible nor incompatible $S-R$ pairings cross the barrier.

There is, however, an alternative, and possibly more convincing, explanation of the phenomenon. In the study by Nicoletti and Umiltà (1984), the two effectors were always the hands and the subjects could have found it easier to represent such effectors by right/left locational codes than by above/below ones. After all, human beings have right and left hands but not upper and lower hands. Since spatial compatibility depends on the coding of the relative spatial locations of stimuli and responses (Nicoletti, Anzola, Luppino, Rizzolatti, \& Umiltà, 1982; Wallace, 1971), it is not surprising that the subjects preferred to use right/left codes to represent the positions of the hands. This preference could have brought about the stronger compatibility effect in the right/left dimension.

This latter explanation can be tested quite easily because there are pairs of effectors for which a coding in terms of above and below is no doubt more natural than is one in terms of right and left. Let us imagine, for example, a spatial compatibility task that requires choosing between a response with the right hand and a response with the left foot. In it, the above/below dimension should become predominant if what matters is the more natural way of 
coding the position of the effectors. By contrast, the hypothesis of the midline barrier would be corroborated if the right/left dimension still proved to be predominant.

The rationale of the series of experiments reported here was the following.

Experiment 1 attempted to replicate the basic finding, that is, the prevalence of right/left locational cues over above/below ones in a spatial compatibility task in which the responses were to be emitted with either the right or left hand. The purpose of Experiment 2 was to demonstrate the spatial compatibility effect when the subject responded with hand and foot and to obtain an estimate of the size of the above/below compatibility effect when right/left cues were absent. Experiment 3 was the crucial one. In it, as in Experiment 1, the right/left and above/below dimensions were varied orthogonally and the subjects responded to right/left and top/bottom stimuli with right/left and top/bottom effectors. This time, however, the bottom effectors were the feet. It was reasoned that if the determinant for prevalence in spatial compatibility was the more natural way of coding the effectors, a stronger spatial compatibility effect should be found for the above/below dimension. By contrast, if the determining factor was the midline barrier, then right/left pairings should still yield a comparatively stronger compatibility effect.

\section{EXPERIMENT 1}

Experiment 1 was essentially a repetition of Nicoletti and Umiltà's (1984) Experiment 4, which had shown that the degree of spatial compatibility was higher for right/left than for above/below positional cues. The main feature of the present experiment was that the two dimensions (i.e., right/left and above/below) were varied orthogonally. We expected both dimensions to be effective in mapping stimuli to responses, thus yielding reliable compatibility effects, and the right/left compatibility effect to be stronger than the above/below one, this in spite of the fact that, as in the previous study, only above/below positional cues were mentioned in the instructions.

\section{Method}

Subjects. Eight 19-25-year-old students served as paid subjects. They were all right-handed, had normal or corrected-to-normal visual acuity, and were naive as to the purpose of the experiment.

Apparatus. The experiment took place in a sound-attenuated cubicle. The subject sat in front of a panel with two light-emitting diodes (LEDs), and the distance between the eyes and the fixation point (a $1^{\circ} \times 1^{\circ}$ black patch in the center of the panel) was kept fixed at $100 \mathrm{~cm}$ through the use of a head- and chinrest. With his or her hands, the subject held two cylinders, each of which had a pushbutton on top. These cylinders were fastened onto two shafts that had different heights and were located about $85 \mathrm{~cm}$ from the panel. The LEDs could be positioned $27^{\circ}$ to the left or right and $27^{\circ}$ above or below the fixation point. Correspondingly, the response device could be $22 \mathrm{~cm}$ to the left or right of the body midline and $22 \mathrm{~cm}$ above or below a conventional center point. Thus, for both light stimuli and response devices there were four possible positions: top right, top left, bottom right, and bottom left. Figure 1 shows a schematic drawing of the experimental setup.
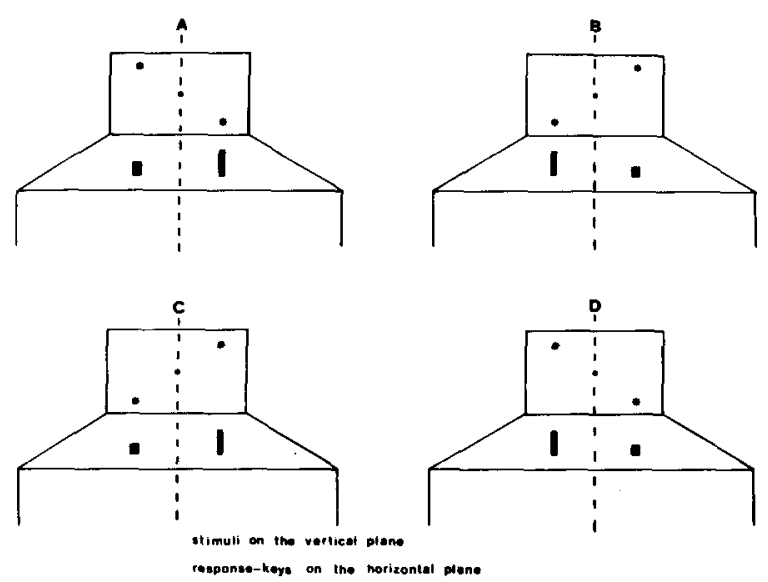

Figure 1. Schematic drawing of stimulus display and response keys for Experiment 1. Panels $A$ and $B$ depict two experimental conditions that were compatible for the right/left dimension and incompatible for the above/below dimension, or vice versa. Panels $\mathrm{C}$ and D depict two conditions that were either compatible or incompatible for both dimensions. In Experiment 3, the arrangement was similar, but the bottom key was operated by the foot.

The apparatus was indirectly illuminated from above. The luminance of the ambient light was about $6 \mathrm{~cd} / \mathrm{m}^{2}$; that of the light stimuli (about $.45^{\circ}$, with a duration of $100 \mathrm{msec}$ ) was about $32 \mathrm{~cd} / \mathrm{m}^{2}$. Interval timing was achieved using interval generators, and response latencies were recorded to the nearest millisecond by an electronic counter that was started with the onset of the light stimulus and stopped by the switch press.

Procedure. The instructions mentioned only above/below positions, and the experimenter purposely avoided any mention of right/left positions. This was possible because any relevant spatial relation could be described unambiguously in terms of either the above/below or the right/left dimension. In other words, both pairs of positional cues were perfectly predictive of a correct response.

There were four experimental conditions, in each of which only two of the four possible positions of stimuli and responses were used. In the first, the two S-R pairings were compatible for both dimensions. For example, when the top right light was shown, the subject was to respond with the higher, right hand, and when the bottom left stimulus was on, the subject was to respond with the lower, left hand. The second condition comprised S-R pairings that were compatible only for the right/left dimension. For example, the top right light required a response with the lower right hand, and the bottom left light required a response with the upper left hand. The third condition was compatible for the above/below dimension only. The top right light required a response with the top left hand, and the bottom left light, a response with the bottom right hand. Finally, the fourth condition was compatible for neither dimension. The top right light required a response with the bottom left hand, and the bottom left light, a response with the top right hand. The above examples describe the $S-R$ pairings used in half of the trials. In the other half, the top left and bottom right positions were used.

There were two experimental sessions on consecutive days, and the first one was preceded by several informal practice trials. In each session, the subjects were given eight blocks of 40 trials, that is, two consecutive blocks for each experimental condition. An acoustic warning signal was delivered 1 sec before stimulus onset, and from the warning signal to the execution of the response, the subject was required to maintain his or her gaze on the fixation point. The interstimulus interval was $5 \mathrm{sec}$.

The assignment of the right or left hand to the top or bottom position and the assignment of the top or bottom light to the right or 
left position were counterbalanced within subjects and across blocks. In the first condition, for example, one block required responding to the top light on the right side with the top hand on the right side and responding to the bottom light on the left side with the bottom hand on the left side. In the other block, the top left light was paired with the top left hand and the bottom right light was paired with the bottom right hand. The order of conditions was counterbalanced between subjects and sessions.

The instructions stressed both speed and accuracy but, with the exception of the practice trials, no feedback was given to the subject. Errors were discarded and replaced. In each block, the two stimuli were shown according to a quasi-random sequence that allowed a maximum of three consecutive presentations in the same position. Thus, after a series of three identical stimuli, the fourth became perfectly predictable; hence, these responses too were discarded and replaced.

\section{Results and Discussion}

Errors were rather rare (about 3.5\%) and were not submitted to statistical analysis. The correct RTs (see Table 1) were entered into a four-way within-subjects analysis of variance with the following factors: vertical position of the light (top or bottom), horizontal position of the light (right or left), vertical position of the hand (top or bottom), and horizontal position of the hand (right or left).

There were two significant main effects. The bottom light was responded to faster than the top light [310 vs. $321 \mathrm{msec} ; \mathrm{F}(1,7)=6.05, \mathrm{p}<.05]$, and the right hand was faster than the left [309 vs. $322 \mathrm{msec} ; F(1,7)=$ $14.61, \mathrm{p}<.01]$. Of greater interest were the significant interactions between vertical position of light and hand $[\mathrm{F}(1,7)=14.23, \mathrm{p}<.01]$ and between horizontal position of light and hand $[F(1,7)=45.55, p<.001]$, which showed two clear-cut compatibility effects. In the vertical dimension, the top hand was $13 \mathrm{msec}$ faster than the bottom hand in responding to the top light (314 vs. $327 \mathrm{msec}$ ), whereas the bottom hand was $11 \mathrm{msec}$ faster than the top hand in responding to the bottom light ( 305 vs. $316 \mathrm{msec}$ ). These differences were shown by every subject, and their reliability was confirmed by pairwise comparisons (Newman-Keuls method; $p<.05$ in both cases). In the horizontal dimension, the right hand responded $57 \mathrm{msec}$ faster than the left to the right-side light (286 vs. $343 \mathrm{msec}$ ), whereas the left hand was $31 \mathrm{msec}$ faster than the right for the left-side light ( 301 vs. $332 \mathrm{msec}$ ). Again, these differences were shown by

\section{Table 1}

Mean Latencies (RTs in Milliseconds) and Standard Deviations (SDs) in Experiment 1 as a Function of Light Position (Top or Bottom and Right or Left), Response Position (Top or Bottom), and Responding Hand or Response Position (Right or Left)

\begin{tabular}{|c|c|c|c|c|c|c|c|c|}
\hline & \multicolumn{4}{|c|}{ Right Light } & \multicolumn{4}{|c|}{ Left Light } \\
\hline & \multicolumn{2}{|c|}{ Top } & \multicolumn{2}{|c|}{ Bottom } & \multicolumn{2}{|c|}{ Top } & \multicolumn{2}{|c|}{ Bottom } \\
\hline & Mean & SD & Mean & SD & Mean & $\mathrm{SD}$ & Mean & SD \\
\hline \multicolumn{9}{|c|}{ Right Hand (or Right Response) } \\
\hline Top & 286 & 18 & 278 & 13 & 326 & 19 & 334 & 28 \\
\hline Bottom & 302 & 16 & 280 & 21 & 339 & 22 & 327 & 19 \\
\hline \multicolumn{9}{|c|}{ Left Hand (or Left Response) } \\
\hline Top & 347 & 12 & 345 & 22 & 298 & 14 & 306 & 23 \\
\hline Bottom & 356 & 35 & 324 & 26 & 310 & 16 & 289 & 11 \\
\hline
\end{tabular}

Table 2

Experiments 1, 2, and 3: Mean Latencies (RTs in Milliseconds) and Standard Deviations (SDs) as a Function of the Main Experimental Conditions

\begin{tabular}{|c|c|c|c|c|c|c|c|}
\hline \multicolumn{2}{|c|}{ B } & \multicolumn{2}{|c|}{$\mathrm{H}$} & \multicolumn{2}{|c|}{ V } & \multicolumn{2}{|c|}{$\mathbf{N}$} \\
\hline Mean & SD & Mean & SD & Mean & $\mathrm{SD}$ & Mean & SD \\
\hline \multicolumn{8}{|c|}{ Experiment 1} \\
\hline 288 & 18 & 299 & 22 & 331 & 22 & 344 & 29 \\
\hline \multicolumn{8}{|c|}{ Experiment 2} \\
\hline - & - & - & - & 339 & 31 & 395 & 55 \\
\hline \multicolumn{8}{|c|}{ Experiment 3} \\
\hline 293 & 32 & 296 & 35 & 343 & 33 & 348 & 50 \\
\hline
\end{tabular}

Note-B: Compatible for both dimensions; $H$ : Compatible for the horizontal dimension only; $V$ : Compatible for the vertical dimension only; $N$ : Compatible for neither dimension.

every subject and were confirmed by pairwise comparisons (both ps $<.01$ ).

Overall, compatibility for the horizontal dimension was significantly stronger than that for the vertical dimension [44 vs. $12 \mathrm{msec} ; \mathrm{F}(1,7)=11.53, \mathrm{p}<.025]$. This was true for every subject.

An additional analysis of variance that compared the mean RTs of the four main experimental conditions (see Table 2) was carried out. The only within-subjects factor was type of condition, whose levels were: pairings compatible for both dimensions, compatible only for the horizontal dimension, compatible only for the vertical dimension, and compatible for neither dimension. This factor showed a significant effect due to type of pairing $[F(3,21)=32.09, p<.001]$. A set of pairwise comparisons confirmed that the four experimental conditions differed from one another (all ps $<.05$ or better).

This experiment was clearly successful in replicating the results of the study by Nicoletti and Umiltà (1984), which had shown the right/left prevalence in spatial compatibility. Both above/below and right/left locational cues were effective in bringing about spatial compatibility, but the effect was much greater for $S-R$ pairings in the horizontal dimension. It appears that the position of the light and that of the hand were coded with reference to both dimensions, but, although the instructions were couched exclusively in terms of top/bottom positions, the outcome of the right/left coding was more effective than the other for mapping stimuli to responses.

The two main effects were not expected and do not seem to be relevant to the main purpose of the experiment. The faster responses to the bottom than to the top light might be due to a greater sensitivity of the upper than of the lower hemiretinae (see Haines \& Gilliland, 1973; Payne, 1967), and the fact that the subjects were all right-handed could explain why the right hand was faster than the left.

\section{EXPERIMENT 2}

Before testing whether the right/left prevalence in spatial compatibility would disappear, or even reverse, with other effectors, an intermediate step was necessary. There 
is no evidence in the literature concerning spatial compatibility when the responses are emitted by hand and foot. Even though one cannot see any reason why spatial compatibility should be confined to the hands, we felt it was appropriate to demonstrate that it occurs also in the case of other pairs of effectors.

Experiment 2 was therefore essentially a repetition of those experiments that had shown the above/below spatial compatibility, with the only notable difference being that the responses were emitted with either the hand or the foot.

\section{Method}

Subjects. Ten new subjects, selected as before, took part in this experiment.

Apparatus. The apparatus was essentially identical to that described for Experiment 1. The response locations were, however, exactly aligned with the midline of the subject's body and the midline of the display panel. The top response device (i.e., that operated by the hand) was $100 \mathrm{~cm}$ above the bottom one (i.e., that operated by the foot). Correspondingly, the light stimuli were positioned $27^{\circ}$ above or below the fixation point and exactly aligned with the body midline. See Figure 2 for a schematic drawing of the S-R arrangement.

Procedure. There were two experimental sessions on consecutive days, the first of which was preceded by informal practice trials. Each session comprised two blocks of 80 trials. The two lights appeared above or below the fixation point according to a quasi-random sequence with a maximum of three consecutive identical presentations. In each session, there were both compatible and incompatible S-R pairings. In the block of compatible trials; the subject responded to the top light with the hand and to the bottom light with the foot; in the incompatible block, the assignment was reversed. The order of compatible and incompatible blocks was counterbalanced across sessions and subjects. Four subjects used the right hand and the right foot; the other four used the left effectors. In all other respects, the procedure replicated Experiment 1.

\section{Results and Discussion}

There were few errors (less than $4 \%$ ), and these were not analyzed. The correct mean RTs (see Table 3 ) were submitted to a two-way analysis of variance with light position (top or bottom) and type of effector (hand or foot) as within-subjects factors. The type of effector main ef-

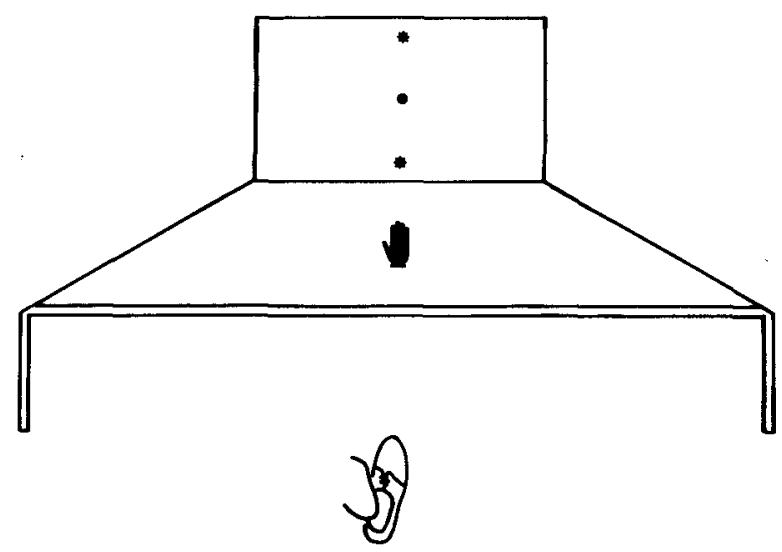

Figure 2. Schematic drawing of stimulus display and response keys for Experiment 2. Here, as in Experiment 3, the hand operated the top key and the foot operated the bottom key.
Table 3

Mean Latencies (RTs in Milliseconds) and Standard Deviations (SDs) in Experiment 2 as a Function of Light Position (Top or Bottom) and Effector (Hand or Foot) or Response Position

(Top or Bottom)

\begin{tabular}{ccccc}
\hline \multicolumn{2}{c}{ Top Light } & & \multicolumn{2}{c}{ Bottom Light } \\
\cline { 2 - 3 } Mean & SD & Mean & SD \\
\hline \multirow{3}{*}{331} & \multicolumn{2}{c}{ Hand (or Top Response) } \\
& 23 & 373 & 54 \\
417 & Foot (or Bottom Response) & \\
4 & 43 & 348 & 34 \\
\hline
\end{tabular}

fect and the interaction were significant $[F(1,9)=16.23$, $\mathrm{p}<.005$, and $F(1,9)=21.89, \mathrm{p}<.005$, respectively]. The light position main effect was only close to significance $[F(1,9)=3.47, p<.1]$.

The two main effects do not seem to be of much interest. The fact that the hand was faster than the foot $(352$ vs. $383 \mathrm{msec}$ ) could well be attributable to biomechanical reasons, and the marginally shorter RTs for the bottom light (360 vs. $374 \mathrm{msec}$ ) could be explained, as before, in terms of a greater sensitivity of the upper hemiretinae.

Of greater importance was the significant interaction that showed a clear-cut spatial compatibility effect. The hand was $\mathbf{4 2} \mathrm{msec}$ faster for the top than for the bottom light ( $331 \mathrm{vs.} 373 \mathrm{msec}$ ), and the foot was $69 \mathrm{msec}$ faster for the bottom than for top light ( $348 \mathrm{vs} .417 \mathrm{msec})$. These effects were present for every subject and proved significant when tested by the Newman-Keuls method (both ps $<.01)$.

A second analysis of variance with repeated measures was performed with type of pairing (compatible or incompatible) as the only factor (see Table 2). It confirmed that compatible RTs were faster than incompatible ones $[F(1,9)$ $=21.86, \mathrm{p}<.005$ ]. In brief, there can be no doubt that above/below spatial compatibility was also present when the subjects used hand and foot for responding. Hence, the undertaking of Experiment 3 was fully justified.

\section{EXPERIMENT 3}

Experiment 3 was aimed at testing whether the above/below compatibility effect would become prevalent when the response required a choice between two effectors that were "naturally" located one above the other (i.e., hand and foot). Thus, except for the effectors used, Experiment 3 replicated Experiment 1.

\section{Method}

Subjects. Ten new subjects, selected as before, participated in the experiment.

Apparatus. The apparatus was identical to that employed in Experiment 1 , except that the bottom response device was moved downward and was now $100 \mathrm{~cm}$ below the top one, so that it could be operated by the subject's foot as in Experiment 2. For both stimuli and responses there were four possible positions: top right, top left, bottom right, and bottom left. Note that the two top responses were emitted by the hands, and the two bottom responses were emitted by the feet. Figure 1 also applies to this experiment if one keeps 
in mind that the two response positions were more distant along the vertical dimension.

Procedure. The procedure was identical to that employed in Experiment 1 , except that now the right foot was used in place of the right bottom hand and the left foot was used in place of the left bottom hand. Of course, the instructions were always in terms of top/bottom positions.

\section{Results and Discussion}

The errors (about 3\%) were not analyzed. The correct RTs (see Table 4) were submitted to a four-way withinsubjects analysis of variance with the same factors listed for Experiment 1.

There were three significant, but scarcely interesting, main effects, which in part confirmed what had been found in the preceding experiments. The bottom light was responded to faster than the top one [313 vs. $326 \mathrm{msec}$; $\mathrm{F}(1,9)=20.59, \mathrm{p}<.005]$, the hand proved to be faster than the foot $[307$ vs. $333 \mathrm{msec} ; F(1,9)=42.17$, $\mathrm{p}<.001]$, and the right-side light was responded to faster than the left-side light $[315$ vs. $325 \mathrm{msec} ; F(1,9)=6.26$, $\mathrm{p}<.05]$.

The two more interesting outcomes of the analysis were the significant interaction between horizontal position of lights and responses $[F(1,9)=37.18, p<.001]$ and the nonsignificant interaction between vertical position of lights and responses $(F<1)$. The first showed that the right-side effector responded $60 \mathrm{msec}$ faster than the left to the right-side light ( $287 \mathrm{vs} .347 \mathrm{msec}$ ) and the left-side effector was 42 msec faster than the right in responding to the left-side light ( 302 vs. $344 \mathrm{msec}$ ). This interaction was present in every subject, and the reliability of the two simple main effects was confirmed by pairwise comparisons (ps < .01). In contrast, the speed of response in the vertical dimension did not seem to depend on the spatial relations beween stimuli and effectors. The bottom effector (foot) was faster for the bottom than for the top light ( $324 \mathrm{vs.} 341 \mathrm{msec}$ ), but the same was also true of the top effector (hand; 302 vs. $312 \mathrm{msec}$ ).

Table 4

Mean Latencies (RTs in Milliseconds) and Standard Deviations (SDs) in Experiment 3 as a Function of Light Position (Top or Bottom and Right or Left), Response Position (Top or Bottom and Right or Left) and Effector (Hand or Foot)

\begin{tabular}{|c|c|c|c|c|c|c|c|c|}
\hline & \multicolumn{4}{|c|}{ Right Light } & \multicolumn{4}{|c|}{ Left Light } \\
\hline & \multicolumn{2}{|c|}{ Top } & \multicolumn{2}{|c|}{ Bottom } & \multicolumn{2}{|c|}{ Top } & \multicolumn{2}{|c|}{ Bottom } \\
\hline & Mean & $\mathrm{SD}$ & Mean & $\mathrm{SD}$ & Mean & SD & Mean & SD \\
\hline \multicolumn{9}{|c|}{ Right Response } \\
\hline $\begin{array}{l}\text { Hand } \\
\text { (or Top) }\end{array}$ & 272 & 17 & 266 & 11 & 335 & 38 & 338 & 53 \\
\hline $\begin{array}{l}\text { Foot } \\
\text { (or Bottom) }\end{array}$ & 310 & 33 & 298 & 26 & 364 & 31 & 353 & 37 \\
\hline \multicolumn{9}{|c|}{ Left Response } \\
\hline $\begin{array}{l}\text { Hand } \\
\text { (or Top) }\end{array}$ & 340 & 25 & 322 & 37 & 300 & 42 & 283 & 31 \\
\hline $\begin{array}{l}\text { Foot } \\
\text { (or Bottom) }\end{array}$ & 368 & 57 & 344 & 26 & 324 & 21 & 302 & 25 \\
\hline
\end{tabular}

Note-Response position (top or bottom) coincides with type of effector (hand or foot).
The nonsignificant higher order interactions (all Fs $<1$ ) showed that the above results also held true when the two effectors were considered separately.

As in Experiment 1, the mean RTs of the four main experimental conditions (see Table 2) were compared in an additional analysis of variance, which showed an effect of type of pairing $[F(3,27)=23.31, p<.001]$. This time, however, the pairwise comparisons were significant (all ps $<.01$ ) only for those conditions that differed in the compatibility of the horizontal dimension.

Therefore, it seems correct to conclude that in the present experiment there was a clear-cut compatibility effect in the horizontal dimension but no such effect in the vertical dimension. It must be pointed out, however, that the magnitude of the compatibility effects was not markedly different in Experiments 1 and 3: 44 versus $51 \mathrm{msec}$ for the horizontal dimension and 12 versus $4 \mathrm{msec}$ for the vertical dimension.

\section{GENERAL DISCUSSION}

Experiment 1 confirmed that spatial compatibility is greater in the right/left dimension than in the above/below dimension. Two explanations were proposed for this right/left prevalence in spatial compatibility. The first makes reference to a midline barrier that would render the compatible right/left S-R pairings, which do not cross it, easier than incompatible S-R pairings, which do cross it. No such barrier would differentially affect compatible and incompatible S-R pairings in the above/below dimension. The second explanation stresses the salience of the right/left locational codes when the subject uses the hands for responding. The two hands (i.e., the effectors employed in Experiment 1) are no doubt more naturally coded as right and left than as top and bottom. Therefore, the process of the spatial coding of S-R pairings, on which spatial compatibility is known to depend, is likely to be more effective for the right/left than for the above/below dimension.

It seemed that these interpretations could be tested by employing pairs of effectors (i.e., hand and foot) that presumably lend themselves more easily to a representation in terms of above/below locational codes than to one in terms of right/left locational codes. Accordingly, although subjects chose between a response with the right or left hand in Experiment 1, they chose between a response with the right hand or the left foot (or vice versa) in Experiment 3. Note that in both experiments the S-R pairings that were incompatible for right/left also entailed the crossing of the body midline, whereas those compatible for right/left occurred exclusively on one side of the body midline. Thus, if the right/left prevalence depended on the crossing of the midline barrier in the case of incompatible right/left S-R pairings, such prevalence should have been present in both experiments. Note also that, although the more natural way to code the effectors was in terms of right and left positions in Experiment 1, the more natural coding was in terms of above and below positions in Experiment 3. Therefore, if the right/left preva- 
lence found in Experiment 1 was due to the relative salience of one of the two pairs of codes that were available, a prevalence for the above/below dimension would be expected in Experiment 3. Of course, this reasoning rested on the assumption that the above/below spatial compatibility existed when the subject used one hand and one foot for responding. The outcome of Experiment 2 proved this to be true.

The results of Experiment 3 supported the notion of the midline barrier by showing that the right/left prevalence in spatial compatibility was still present even if the effectors involved were naturally positioned one above the other and thus easier to code in terms of above and below positions. However, for reasons that will be explained below, we prefer to be more cautious and to confine ourselves to saying that Experiment 3 showed that the right/left prevalence in spatial compatibility does not depend on the type of effector used.

As for the midline barrier hypothesis, available evidence belies it in its strong version. Spatial compatibility cannot be attributed to the crossing of the body midline, which occurs in the case of incompatible S-R pairings, because it is also present in the vertical dimension (see Experiments 1 and 2 here and Experiments 1 and 4 in Nicoletti \& Umiltà, 1984). Moreover, spatial compatibility was also observed when all S-R pairings were kept within one side in relation to the body midline (see Nicoletti et al., 1982). Therefore, there can be no doubt that spatial compatibility exists irrespective of whether the incompatible S-R pairings cross the body midline. In fact, it is very likely to originate from a correspondence (compatible S-R pairings) or lack of correspondence (incompatible S-R pairings) between the positional codes associated with stimuli and responses (see Nicoletti et al., 1982; Nicoletti, Umiltà, \& Ladavas, 1984; Wallace, 1971).

There is also a weaker version of the midline barrier hypothesis, namely that put forward in the introduction. It states that, although spatial compatibility is due to the coding of the relevant locations, the effect is greater in the right/left dimension than in the above/below dimension because only in the former do the incompatible S-R pairings occur across the body midline. There would be two, presumably additive, factors in spatial compatibility. The first and more important one is the congruence of the positional codes, which brings about spatial compatibility in both the right/left and above/below dimensions. The second is the crossing by incompatible S-R pairings of the body midline, which causes the right/left prevalence. This weaker version of the hypothesis, however, is rendered scarcely tenable by the finding that in the right/left dimension spatial compatibility is more or less of the same magnitude regardless of whether the incompatible $S-R$ pairings occur across the body midline (see Nicoletti et al., 1982). In contrast, spatial compatibility should accordingly be stronger when the incompatible S-R pairings cross the midline and the two factors thus combine their effects. In conclusion, even though the right/left prevalence is also present when it is the foot that emits one of the responses, an explanation of the phenome- non based on the notion of the midline barrier seems unlikely.

Why then is spatial compatibility stronger in the right/left dimension? One can speculate that for a horizontally symmetrical organism, discriminating between right and left is a rather difficult task (see, e.g., Corballis \& Beale, 1976), as it also is at a perceptual level (see Farrell, 1979). Because of this, attention might be allocated automatically to the right/left dimension, thus rendering right/left locations more salient. This interpretation is similar to that proposed for the so-called visual dominance (see, e.g., Posner, Nissen, \& Klein, 1976). Human beings are thought to allocate attention to the visual modality at the expense of other modalities because visual stimuli are more difficult to detect.

Assuming that the magnitude of compatibility effects is determined by, among other things, the salience of the relevant positional cues, an attentional bias in favor of right/left cues might be the cause of the right/left prevalence in spatial compatibility. The fact that above/below spatial compatibility is much stronger when only above/below cues are available (see Experiment 2 here and Experiment 1 in Nicoletti \& Umiltà, 1984) than when right/left cues are also present (see Experiments 1 and 3 here and Experiment 4 in Nicoletti \& Umiltà, 1984) certainly supports this, as yet speculative, explanation.

\section{REFERENCES}

BRUNer, J. S. (1971). The growth and structure of skill. In K. J. Conolly (Ed.), Motor skills in infancy. New York: Academic Press.

Corballis, M. C., \& BeALE, I. L. (1976). The psychology of left and right. Hillsdale, NJ: Erlbaum.

FARRELl, W. S. (1979). Coding left and right. Journal of Experimental Psychology: Human Perception and Performance, 5, 42-51.

Haines, R. F., \& Gilliland, K. (1973). Response time in the full visual field. Journal of Applied Psychology, 58, 289-295.

Maki, R. H., Grandy, C. A., \& HaUge, G. (1979). Why is telling right from left more difficult than telling above from below. Journal of Experimental Psychology: Human Perception and Performance, $5,52-67$

Maki, R. H., MaKI, W. S., \& Marsh, L. G. (1977). Processing locational and orientational information. Memory \& Cognition, 5, $602-616$.

Nicoletti, R., Anzola, G. P., Luppino, G., Rizzolatti, G., \& UMILTÁ, C. (1982). Spatial compatibility effects on the same side of the body midline. Journal of Experimental Psychology: Human Perception and Performance, 8, 664-673.

NicoletTI, R., \& UmiLtì, C. (1984). Right-left prevalence in spatial compatibility. Perception \& Psychophysics, 35, 333-343.

Nicoletti, R., Umiltà, C., \& Ladavas, E. (1984). Compatibility due to the coding of the relative position of the effectors. Acta Psychologica, 57, 133-143.

Payne, W. H. (1967). Visual reaction times on a circle about the fovea. Science, 155, 481-482.

Posner, M. I., Nissen, M. J., \& Klein, R. (1976). Visual dominance: An information-processing account of its origins and significance. Psychological Review, 83, 157-171.

SHOLL, M. J., \& EGETH, H. E. (1981). Right-left confusion in the adult: A verbal labeling effect. Memory \& Cognition, 9, 339-350.

Simon, J. R. (1969). Reactions toward the source of stimulation. Journal of Experimental Psychology, 81, 174-176.

WAllace, R. J. (1971). S-R compatibility and the idea of a response code. Journal of Experimental Psychology, 88, 354-360.

(Manuscript received April 3, 1985; revision accepted for publication August 9, 1985.) 